\title{
Aspectos sobre el tratamiento quirúrgico del cáncer epitelial de ovario
}

\author{
Gildardo Gallego Noreña*
}

RESUMEN: El cáncer de ovario causa más muertes en las mujeres que otros tumores ginecológicos. La incidencia en países desarrollados como Japón y Estados Unidos es elevada; en nuestro país ocupa el segundo o tercer lugar.

La cirugía para cáncer de ovario es una de las más difíciles que realiza el ginecólogo. Al planear el tratamiento de una paciente con cáncer de ovario se debe realizar una preparación integral de ella.

La historia clínica de la paciente debe ser excelente haciendo énfasis en los antecedentes personales y familiares.

Es importante la clasificación clínica, y agrupar las pacientes en categorías de alto y bajo riesgo con el fin de definir el tratamiento quirúrgico a seguir.

Al iniciar la cirugía el oncólogo debe tener unas normas fijas de procedimiento como son: incisión vertical, lavado peritoneal para citología, exploración abdominal completa, remoción del tumor intacto, del ovario contralateral, útero, trompas de Falopio, epiplón y biopsias aleatorias peritoneales. Se comenta las indicaciones de una laparatomía de segunda mirada.

PALABRAS CLAVES: Cáncer de ovario, omentectomía, citorreducción.

SUMMARY: The ovarian cancer cause more deaths in the women than other ginecology tumors. The incidence in U.S.A. and Japan is high, in our country is the second or thirth place.

The surgery por ovarian cancer is the most difficult that ginecologist perform. When we plan treatment of patients with ovarian cancer is necesary a correct integral preparation of them. The clinic record will be excellent, with emphasis in familiar and personal antecedents.

The clinical clasification is very important to agroupe the patients and define the surgical treatment.

Two categories are announce: the higt and low risk, this article gives the caracters to inclsude the patiens.

In the moment to perform a surgery the oncologist would have certain conditions to begin the procedure: vertical incision, peritoneal washing for citology, complete abdominal exploration remotion intact tumors, remotion contralateral ovary, uterine, and falopia tuba, remotion of ommentun and aleatory peritoneal biopsias.

The indication for a second look laparatomy and the laparoscopy surgery por ovarian cistic are comented.

KEY WORDS: Ovarian cancer, omentectmie, citoreduction.

\section{Introducción}

El cáncer de ovario causa más muertes en la mujer que otros tumores ginecológicos en USA. Tiene una incidencia elevada en países desarrollados como Japón y Estados Unidos, en este país se diagnostican 22.000 casos nuevos por año y ocurren 13.000 muertes por esta causa, además un 26 por ciento (3.000 casos) son estadíos I y II, el resto estados avanzados. El pico de incidencia según edad está en la quinta y sexta década de la vida.

En nuestro medio la incidencia es menor, ocupa el tercero o cuarto lugar, específicamente en Medellín en la Clínica de Tumores del Hospital San Vicente de Paúl en

Coordinador y Profesor. Grupo de Oncología Ginecológica. Departamento de Obstetricia y Ginecología. Facultad de Medicina. Universidad de Antioquia. un período de 10 años (1985-1994) se tienen registradas 190 pacientes y 102 de éstas son menores de 50 años.

Los tipos histológicos más frecuentes son los tumores epiteliales que constituyen 85 al $90 \%$, el resto son subtipos germinales y de células del estroma.

El cáncer de ovario debe considerarse enfermedad del peritoneo de la cavidad abdominal, pues cualquier superficie de ésta serosa está en riesgo de ser asiento de metástasis microscópicas. La propagación puede ir a ganglios retroperitoneales y aunque en ocasiones es predecible, muchas veces existe variación en el comportamiento de los tumores.

El tratamiento del cáncer de ovario se apoya en tres pilares básicos:

1. Cirugía

2. Quimioterapia

3. Radioterapia 
Este artículo se referirá únicamente al tratamiento quirúrgico

El cirujano que realiza el tratamiento del cáncer de ovario no sólo debe extirpar la mayor cantidad de masa tumoral posible sino también evaluar la extensión de la enfermedad.

La cirugía para el carcinoma de ovario es uno de los tratamientos más difíciles que realiza el ginecólogo, este último tiene responsabilidad muy importante en definir las características de la presentación del tumor, determinar su verdadera extensión y consignar al final si deja tumor residual.

El cáncer de ovario es muy raro en la paciente embarazada, la incidencia varía de 1 en 12.000 a 1 en 15.000 embarazos, con promedio de 1 en 25.000. Son más frecuentes los tumores benignos tales como quistes serosos simples y teratomas quísticos. Al intervenir una paciente embarazada con tumor de ovario se debe considerar el tiempo de gestación y la sintomatología que produce; además, realizar un buen diagnóstico diferencial. Si se diagnostica un carcinoma de ovario en una paciente embarazada en el primero y segundo trimestre se procede a tratarlo siguiendo el protocolo de manejo usual haciendo caso omiso del embarazo y si se diagnostica en el tercer trimestre se prefiere alcanzar la madurez fetal para intervenir el carcinoma de ovario (1).

El Tratamiento quirúrgico persigue tres objetivos fundamentales:

1. Extirpar el tumor voluminoso para aliviar los síntomas,

2. Evaluar el grado de propagación más allá del ovario y

3. Practicar la cirugía citorreductora

\section{Preparación de la paciente}

Al planear el tratamiento se debe valorar y preparar la paciente, analizar aspectos emocionales, sicológicos y fisiológicos. Debe estar enterada de los aspectos y consecuencias más importantes de su enfermedad tales como cirugías radicales y la posibilidad de cirugías únicamente exploradoras en las cuales no se puede extirpar ningún tumor debido a lo diseminado del mismo. En pacientes jóvenes es necesario conocer los deseos de paridad de la misma.

La historia clínica será lo más cuidadosa posible, haciendo énfasis en diagnósticos diferenciales en los sistemas urinarios, pélvicos, descartar padecimientos diverticulares, procesos anexiales, enfermedad inflamatoria pélvica, adherencias pélvicas que pueden dificultar el examen y vías de acceso, además consignar si ha tenido cirugías pélvicas previas. Es necesario recordar que el $70 \%$ de las mujeres con cáncer de ovario se presenta en estados avanzados III y IV en los cuales la cirugía sola ya no tiene un papel único en la curación sino que se debe apoyar en la quimioterapia.

Los antecedentes personales y familiares son de gran importancia. Las pacientes con historia familiar positiva de cáncer de endometrio, mama y colon tienen mayor riesgo de padecer malignidad pélvica o cáncer de ovario.

Se recomienda realizar mamografía preoperatoria, en pacientes mayores de 40 años, ya que algunas pacientes con cáncer de ovario tienen mayor riesgo de desarrollar un tumor de mama. Un cáncer de ovario puede representar metástasis ocultas de un cáncer mamario. Se debe pedir: perfil hematológico que incluya hemograma, electrólitos, úrea, creatinina, función hepática, citoquímico de orina y antígeno Ca 125 .

Ante una tumoración anexial persistente se debe pedir estudios de imagenología, tomografía axial computarizada, ecografía o resonancia magnética. Es necesario preparación del intestino con enemas previos a la cirugía y en algunos casos se aconseja el uso de heparina para disminuir riesgo de trombosis venosa profunda y embolia pulmonar.

\section{Clasificación clínica}

La clasificación por etapas constituye un sistema quirúrgico patológico modificado por la FIGO en 1985 (2). El principal cambio en la clasificación de la Figo en 1985 es la agregación de subetapas dentro de la etapa III con base en la evolución natural y la extensión del tumor metastásica (3).

En un estudio del M.D. Anderson donde clínicamente se clasificaron 100 pacientes con estados I y II se les realizó reclasificación quirúrgica se demostró que 31 pacientes tenían enfermedad más avanzada que la notada en la clasificación inicial (2). Argumento a favor de una buena clasificación quirúrgica.

La etapa de la enfermedad, está determinada por la extensión del tumor en el momento del diagnóstico inicial.

\section{Clasificación 1985}

Estado I: enfermedad limitada a los ovarios

I A: limitado a un ovario, no ascitis, no tumor en la superficie, cápsula íntegra.

$I B$ : limitado a los dos ovarios, no ascitis, no tumor en la superficie, cápsula íntegra.

I C: tumor I A o B con tumor en la superficie de uno o ambos ovarios, cápsula rota; o ascitis con células malignas o lavado peritoneal positivo.

Estado II: tumor que compromete uno o ambos ovarios y tiene extensión pélvica.

II A: extensión y/o metástasis útero y/o trompas

II $B$ : extensión a otros tejidos pélvicos

II C: estado II A o B con tumor en la superficie de uno o ambos ovarios, cápsula rota; o ascitis con células malignas o lavado peritoneal positivo.

Estado III: tumor que compromete uno o ambos ovarios con implantes peritoneales extrapélvicos y/o ganglios retroperitoneales positivos. Metástasis hepáticas superficiales. Tumor limitado a la pelvis pero con extensión histológicamente comprobada a intestino delgado o epiplón.

III A: tumor limitado a la pelvis, pero con extensión microscópica histológicamente comprobada a la superficie peritoneal abdominal.

III B: tumor que compromete uno o ambos ovarios con implantes histológicamente confirmados en superficies peritoneales abdominales que no exceden los dos centímetros de diámetro mayor. Ganglios negativos.

III C: implantes abdominales mayores de dos centímetros. Ganglios retroperitoneales o inguinales positivos. 
Estado IV: tumor que compromete uno o ambos ovarios con metástasis a distancia. Derrame pleural con evidencia de células malignas. Metástasis hepáticas intraparenquimatosas (4).

\section{Protocolo de manejo}

El tratamiento quirúrgico se debe apoyar en una clasificación clínico quirúrgica bien documentada. Desde el punto de vista quirúrgico se normatiza el siguiente protocolo para obtener un buen estadiaje de la entidad: (2)

1. Incisión vertical

2. Lavados peritoneales múltiples para citología

3. Remoción del tumor intacto

4. Exploración abdominal completa

5. Remoción del ovario remanente, útero y trompa

6. Omentectomía

7. Muestreo ganglionar pélvico y ganglios sospechosos, localizados en cavidad abdominal.

8. Biopsias peritoneales al azar o aleatorias de: diafragma, repliegue vesical, goteras parietocólicas y cualquier área sospechosa. La biopsia por congelación puede apoyar la clasificación.

9. Apendicectomía: en estados iniciales para descartar enfermedad oculta.

Incisión: debe ser vertical en línea media supra e infraumbilical desde apéndice xifoides hasta la sínfisis del pubis que permita tener acceso a la parte superior del abdomen para visualizar el diafragma, la superficie hepática y palpar ganglios paraórticos. En ciertos casos es necesario seccionar el ligamento triangular o suspensorio del hígado. No se pueden hacer consideraciones estéticas en una paciente en quien se sospecha cáncer de ovario.

La exploración quirúrgica documenta el grado de tumor residual usado para establecer etapas de la FIGO e identifica áreas de riesgo que son susceptibles de biopsias.

En ciertos casos se puede usar el laparoscopio a través de la laparotomía para sitios que son inaccesibles a la exploración visual o manual.

Omentectomía: el epiplón está constituido por dos capas pegadas de peritoneo que se originan en la cara mayor del estómago y suspenden del colon transverso a través de un plano vascular.

El omento es asiento de micrometástasis (5) por lo tanto se justifica su extirpación, es el tratamiento estándar. Además, si este tiene metástasis puede existir propagación a curvatura mayor del estómago, hemidiafragma o el bazo.

En algunos centros que usan isótopos radiactivos intraperitoneales piensan que una vez extirpado el epiplón la cantidad del isótopo que se distribuye sobre la superficie peritoneal es mayor.

Vías de acceso retroperitoneal: son útiles para obtener acceso quirúrgico hacia ganglios linfáticos superiores y pélvicos. Se practican en peritoneo lateral de colon ascendente y descendente, permiten palpación, inspección y extirpación de masas tumorales o de ganglios preaórticos o precava.

Apendicectomía: Se sabe que el adenocarcinoma del apéndice es raro y en ocasiones es difícil distinguirlo del cáncer de ovario. De otra parte se ha demostrado que ésta estructura puede ser asiento de micrometástasis (5).

Resección intestinal y colostomía: se acepta cuando existe una lesión localizada o cuando la enfermedad residual es sensible a la quimioterapia. En algunos casos seleccionados se puede realizar resección parcial de vejiga.

Cirugía citorreductora: el principio mayor en el enfoque terapéutico de toda tumoración de ovario es quirúrgico. La cirugía citorreductora da mayor índice de sobrevida cuando deja masas residuales menores de uno o dos centímetros de diámetro y cuando éstas son mayores la sobrevida es menor.

Argumentos:

1. Las pacientes están muy sintomáticas debido a grandes masas tumorales y la remoción de dichas masas mejora el bienestar, el estado nutricional y posiblemente mejora la tolerancia a la quimioterapia.

2. La citorreducción está indicada porque en los tumores muy grandes se disminuye la eficacia de la quimioterapia, no tienen buen aporte sanguíneo, disminuye la fracción de crecimiento y se favorece la presencia de células o clonas resistentes.

Se ha usado el término "Debulking" que significa esculpir a través de las masas tumorales; (7) técnicamente es una cirugía poco elegante.

Una investigación cooperativa del Colegio de Ginecólogos Oncólogos (COG) de Estados Unidos en un período de 10 años analizan 914 pacientes y concluyen que existe una alta probabilidad de muerte cuando las pacientes presentaron las siguientes características: edad mayor de 50 años, estadíos IV, tipo histológicos diferentes al seroso y tumor residual mayor de 2 centímetros de diámetro.

\section{Criterios de riesgo}

Un número de características de la lesión primaria determinan el pronóstico de la paciente. Para asignar Yas pacientes a categorías específicas de riesgo se tienen en cuenta los hallazgos de la laparatomía exploradora siguiendo todos los pasos enumerados en el protocolo de manejo. Se divide en características de bajo y de alto riesgo (2).

\section{Características de bajo riesgo:}

- Grado histológico 1-2 (bien o moderadamente diferenciados)

- Cápsula intacta

- Ningún tumor en la superficie peritoneal externa

- Ausencia de ascitis

- Lavado peritoneal negativo

- Crecimiento confinado a los ovarios

Características de alto riesgo:

- Grado histológico 3 (francamente anaplásico o pobremente diferenciado)

- Cápsula rota

- Tumor en superficie peritoneal externa

- Citología positiva del lavado peritoneal

- Presencia de ascitis

- Crecimiento fuera del ovario. 


\section{Tratamiento quirúrgico}

Etapas I Y II:

Se debe proceder a exploración bimanual bajo anestesia y confirmar la masa tumoral. Clasificar por etapas, con incisión vertical amplia que permita la palpación, inspección y toma de biopsias de vísceras abdominales, ganglios retroperitoneales, superficie diafragmática. Palpación de superficies peritoneales y parietales.

Si hay diseminación obvia de la pelvis por el carcinoma, es casi un estadío III en cambio si no hay diseminación más allá de la pelvis está indicado lavados, citologías y biopsias de peritoneo y retroperitoneo.

Los lavados y citologías se realizan de fondo de saco posterior, goteras parietocólicas derecha e izquierdas y diafragma derecho e izquierdo. La citología puede ser positiva entre un 10 y un $36 \%$ de cánceres en etapas I, puede ser negativas aún en presencia de cáncer manifiesto en un número considerable de casos.

Se debe observar la cápsula del tumor, adherencias, roturas, excrecencias, extirpar el tumor intacto y enviar corte por congelación en caso necesario. El pronóstico empeora con la rotura del tumor, penetración de la cápsula, adherencias a estructuras adyacentes $(8,5,9)$.

Si no es necesario conservar el potencial reproductivo se procede a realizar histerectomía total con salpingooforectomía bilateral, omentectomía y apendicectomía.

En pacientes jóvenes con estadío Ia y deseos de conservar la función reproductiva se procede a realizar laparatomía clasificatoria, anexectomía unilateral con conservación del útero y ovario contralateral. Además, se debe asesorar a la paciente en cuanto a riesgos potenciales, vigilancia cuidadosa y extirpar el ovario residual después de cumplir propósitos de procreación.

\section{Segunda mirada}

Ganó popularidad en la década de 1970 - 1980 y se volvió sistemática para el seguimiento de las pacientes sometidas a quimioterapia por carcinoma de ovario. También ha sido denominada "Second Look" o laparatomía de segunda observación; se refiere a un procedimiento para evaluar la respuesta a la quimioterapia en una mujer sin enfermedad clínica $(7,10)$.

La denominación "sin enfermedad clínica" quiere decir que la exploración física no demuestre datos de cáncer, los estudios de imagenología son negativos y los marcadores tumorales son normales; en otras palabras la paciente debe hallarse potencialmente sin enfermedad con base a una evaluación incruenta exhaustiva.

La laparatomía de segunda mirada debe cumplir requisitos específicos de palpación de cavidad abdominal, toma de muestras de lesiones sospechosas, lavados de todas las goteras y repliegues. Cuando no hay tumor macroscópico después de la exploración exhaustiva se indican biopsia al azar de fondo de saco posterior, peritoneo vesical, goteras, hemidiafragmas, epiplón residual, ganglios aórticos y pélvicos. Algunos autores toman hasta 30 muestras para estudio histológico.

La segunda mirada persigue:
1. Determinar la respuesta al tratamiento de la primera línea de quimioterapia y seleccionar el subsiguiente tratamiento.

2. Definir el pronóstico y

3. Determinar la influencia sobre la sobrevida

Lo más importante es seleccionar las pacientes para estrategias alternativas de tratamiento.

La quimioterapia intraperitoneal es más efectiva en pacientes con enfermedad residual mínima, condición que solo es evaluable con la segunda mirada.

Otra alternativa para la segunda mirada es realizarla con laparoscopia, con esta técnica existe la dificultad de palpar exhaustivamente la cavidad, puede dar falsos negativos de un 20 a un 55\%, porcentaje alto que se debe considerar y evaluar.

Con la introducción de la videolaparoscopia es posible disminuir el número de falsos negativos y permite realizar biopsias de sitios específicos, siempre deberá explorarse el retroperítoneo.

\section{Cirugía laparoscópica de quistes ováricos}

Esta nueva tecnología ha llevado a que muchas pacientes por conservar su abdomen sin incisiones lo soliciten en forma explícita a su médico. Es este último quien debe analizar cuidadosamente el caso, usar criterios estrictos preoperatorios y protocolos de manejo con el fin de decidir cual paciente se beneficia de la cirugía laparoscópica. El método laparoscópico de manejo de un tumor pélvico ofrece el potencial de una cirugía mínimamente cruenta y eficaz.

La aplicación racional de éstas técnicas representa un verdadero avance de la ginecología (11). La no selección cuidadosa de la paciente conduce a la sorpresa desagradable de realizar cirugía translaparoscópica para un cáncer de ovario, por esto es indispensable el uso de criterios preoperatorios y procedimientos transoperatorios estrictos.

Se puede agrupar las pacientes con tumor quístico de ovario para un manejo más racional en pre y postmenopáusicas (12).

Pacientes premenopáusicas: la mayoría de los quistes en estas pacientes son funcionales, el tratamiento es observación dependiendo del tamaño.

$\mathrm{Si}$ se piensa que es quirúrgico se sugiere que la decisión se apoye en criterios que se enunciarán para las postmenopáusicas en las cuales se tienen en cuenta antecedentes personales, familiares, ecografías y marcadores tumorales.

Pacientes muy jóvenes con lesiones significativas de ovario debe determinarse HCG y alfafetoproteína antes de proceder a laparoscopia. Un nivel elevado ligeramente de CA 125 puede sugerir endometriosis. Pacientes con CA-125 menor de $35 \mathrm{U} / \mathrm{ml}$ y tumor pélvico éste generalmente es benigno en el $97 \%$ de los casos independiente de la edad (13).

$\mathrm{Si}$ analizados todos los datos en la paciente premenopáusica se concluye que no existe riesgo se procede a cirugía laparoscópica.

Pacientes postmenopáusicas: en estas pacientes debe de analizarse: 
1. Antecedentes personales y familiares

2. Hallazgos físicos

3. Marcadores tumorales

4. Criterios ecográficos

Antecedentes personales y familiares: se analiza si la paciente presenta un primer grado de consanguinidad relacionado con cáncer de ovario, mama, colon, endometrio. Las pacientes con historia positiva de estos antecedentes tienen más riesgo de malignidad ovárica y requieren mejor Laparatomía. Cualquier dato o antecedente que sugiera cáncer de ovario requiere mejor laparatomía (13).

Pacientes posmenopáusicas con marcadores elevados CA 125 requieren laparatomía.

Ecografía: los tumores benignos, ecográficamente son lesiones únicas, uniloculadas de bordes nítidos, sin excrecencias, no existe componente sólido, no hay líquido en el fondo de Douglas. En algunos casos la ecografía sugiere lesión maligna si presenta, excrecencias, bordes rudos o irregulares, multiloculación, lesiones múltiples, tabiques gruesos, presencia de ascitis y adherencias intestinales (14). Granberg y colaboradores citados por William Parker en un estudio de 94 mujeres posmenopáusicas y 86 mujeres menstruantes encontraron que el número de papilas detectadas por ecografía se correlacionó con la posibilidad de cáncer (15). Es importante recordar que la ecografía sola no servirá para identificar malignidad y se impone sumar otros criterios de riegos ya mencionados.

Pacientes postmenopáusicas con quistes pequeños que reúnan criterios de benignidad se benefician de cirugía translaparoscópica. Algunos autores son partidarios de aspiración transvaginal del quiste guiada por ecografía.

De todas maneras los líquidos aspirados deben ir a estudio citológico. También es necesario recordar que resultados negativos de líquidos no descartan malignidad, pero los hallazgos positivos son una indicación de laparatomía. Se recomienda seguimiento ecográfico en las pacientes que se le ha realizado aspiración de quistes mediante vía transvaginal o translaparoscópica. Cuando se encuentra un cáncer inesperadamente durante una cirugía laparoscópica de un tumor anexial, el cirujano debe estar preparado para proceder con la laparatomía de clasificación por etapas y el tratamiento adecuado (15).

La paciente que se somete a cirugía laparoscópica de un tumor de ovario, debe tener consentimiento informado de que si se encuentra algún dato sugestivo de neoplasia, puede despertarse con laparotomía exploradora.

\section{BIBLIOGRAFIA}

1. Peralta María Teresa, Caro Jorge Eduardo. Cirugía para afección ovárica durante el embarazo, experiencia institucional. Rev Col de Obst y Gin. 1995; 46(1): 61-66.

2. Thigpen Tate, Blessing John, Vance Rallph Lambuth Bruce. Management of patients with stage I and II ovarian carcinoma. Proceeding of the perurgia international Cancer conference II. Perurgia Italy. 1989; 41-49, June 16-17.

3. Soper JT. Tratamiento del cáncer ovárico epitelial en etapa temprana. Clin Obstel y Ginecol 1994; 2: 389-403.

4. Manual de normas de manejo del cáncer genital femenino. Revista Colombiana de Obstetricia y Ginecología. 1993; 18: 28-39.

5. González JM. Oncología Ginecológica. Salvat, Barcelona; 1991; 309-363.

6. Gershenson D. Citorreducción primaria del cáncer ovárico epitelial avanzado. Clínicas de Ginecología y Obstetricia. Temas Actuales. 1994; 1: 121-140.

7. Colombo N., Maggioni A., Calleoni R., Chiari S., Torri W., Mangioni C. Surgery for advanced ovarian Cancer. Proceeding of the Perurgia. International Cancer Conference II Perurgia Italia. 1989; 16-18.
8. Ivor B., Stephen R. Tratamiento del cáncer epitelial en etapas tempranas. Cl Obst y Gin. Temas Actuales. 1994; 1: 107-119.

9. Disaia PJ., Creasman WT. Clinical Gynecologic Oncology. Mosby Year Book, St. Louis Missouri; 1993; 333-423.

10. Clark P., D.L., Kohler, Matthew, Hur Teau Jean, El bendary et al. Cirugía para cáncer de ovario avanzado. Clin Obstet Gynec 1994; 2 : 405-423.

11. Maiman M. Extirpación laparoscópica de tumores anexiales: precauciones. Clin Obst y Gin de Norte América, 1995; 2: 355-364.

12. Seltzer V., Maiman M., Boyce J. Cirugía laparoscópica para atención de quistes ováricos, Mundo Médico. 1993; 41-45.

13. Parker W., Tratamiento laparoscópico de tumores de anexos. Cl Obs Gin. Temas Actuales. 1994; 1: 79-91.

14. Steege, JF. Método laparoscópico en los tumores anexiales. Clin Obstet y Gynecol 1994; 2: 359-371.

15. Parker W. Tratamiento laparoscópico de los tumores anexiales, Clin Obst y Gin de Norte América, 1995; 2: 347-353 